\title{
Effect of Amplifier Gain on Photoacoustic SNR (Signal to Noise Ratio) in an LED-based Photoacoustic Imaging System
}

\author{
Toshitaka Agano, Kunio Awazu \\ Graduate School of Engineering, Osaka University
}

\begin{abstract}
The possibility of using photoacoustic imaging for functional diagnosis has attracted much attention especially in the clinical field. Among such imaging systems, a system, which offers real-time imaging using compact and low-priced LEDs as a light source, has appeared. Compared to solid state lasers, the LED pulse energy was extremely small, so it had been thought that imaging would be extremely difficult, but by adding a pre-amplifier, real time photoacoustic imaging became possible. However the signal-to-noise ratio (SNR) and the amplifier gain needed for making real time imaging possible have remained unclear. The present study was designed to clarify these data. The results showed that, using a tissue phantom and human fingers, an SNR > 4 and amplifier gain $>80 \mathrm{~dB}$ were required, and demonstrated why making an image without a pre-amplifier had proved difficult.
\end{abstract}

Key words: Photoacoustic $\cdot$ LED $\cdot$ Amplifier gain $\cdot$ SNR $\cdot$ image

\section{Introduction}

Photoacoustic imaging is the creation of an image based on the detection of ultrasonic waves emitted upon thermal expansion of incident pulsed light-excited light-absorbing substances. Photoacoustic imaging enables in vivo mapping of photosensitive agents (e.g. ICG etc.) ${ }^{1)}$, detection of hemoglobin ${ }^{2}$, measurements of function such as the degree of oxygen saturation ${ }^{3)}$ and identifying foreign objects such as injection needles or markers. ${ }^{4}$ Anticipation is high, especially in the clinical field for this technology to become a new low-cost and compact modality for functional diagnosis, compared with the presently available MRI.

When a human patient is subjected to photoacoustic imaging, the excitation light will scatter as it enters the tissue and therefore coherence of the light is not a requirement, so in which case a low-cost LED light source may be used instead of solid-state lasers. Although there are few studies on photoacoustic imaging using LEDs as the light source 5, 6), the systems used were not compatible with real-time visualization which would be required for

Addressee for Correspondence:

Toshitaka Agano

(2-1 Yamadaoka, Suita-shi, Osaka 565-0871, Japan)

Tel: +81 668797885 Fax: +81668797363

Corresponding author: toshiaga@yahoo.co.jp clinical application. This study was therefore designed to invistigate the basic technology of photoacoustic imaging, both compact, low-cost and fit for future clinical applications in human patients. ${ }^{7-10)}$

In general, the light sources used in photoacoustic imaging systems are solid-state lasers where the pulse widths are a few ns to several dozen ns, the energy per pulse being a few $\mathrm{mJ}$ to several hundred $\mathrm{mJ}$ and the calculated peak power being 0.3 MW-100 MW. Conversely when LEDs are used as the light source, the pulse-width is $70 \mathrm{~ns}$, energy per pulse is $400 \mu \mathrm{J}$ and the calculated peak power is $0.0057 \mathrm{MW}$ which extremely small compared to that of solid-state lasers ${ }^{11)}$. Although previous studies have been conducted using LEDs as the excitation source, these were to confirm the principles of photoacoustics in the experimental setting, and detection of signals was possible ${ }^{6}$. However, clinical application of these systems where real time visualization and imaging are warranted, was impractical at the time. Recently a pre-amplifier has however been developed and when coupled with the LED source,, it increased the amplifier gain from the normal $40 \mathrm{~dB}$ of a normal solid-state laser excitation photoacoustic imaging system to a significantly higher 97 dB. This Acoustic X (CYBERDYNE: Tokyo, Ja-

Received date: October. 15th, 2018

Accepted date: November, 14th, 2018

J-STAGE Advance Publication Date: December, 4th, 2018 
pan) system has made LED-based photoacoustic imaging possible for the first time.

\section{Purpose}

Although real time imaging using an LED-based photoacoustic imaging system with an amplifier gain of $97 \mathrm{~dB}$ became possible, the relationship between the required signal-to-noise ratio (SNR) and the gain required to achieve that SNR has remained unclear. In the present study, the authors investigated the relationship using a phantom and a human finger. The authors also discussed why real time imaging is difficult when LEDs are used as the light source in a set-up for a normal solid-state laser-based photoacoustic imaging system with an amplifier gain of $40 \mathrm{~dB}$.

\section{Materials and Method}

\subsection{Measurements of the photoacoustic SNR for the imaging of bovine blood}

The experimental set-up is shown in Figure 1. Acoustic $\mathrm{X}$ (CYBERDYNE: Tokyo, Japan) shown in Figure 2(a) was used as the measurement system. In order to detect the photoacoustic signals, the LED arrays were placed on both sides of the detecting ultrasound probe (central frequency 9.5 MHz, bandwidth 80\%, pitch $0.3 \mathrm{~mm}, 128$ channels) as shown in Figure 2(b). The direction of the ultrasound probes $128 \mathrm{ch}$ and the longitudinal direction of the LED arrays are directed at the depth of this page, as shown in Figure Figure 1 and Figure Figure 2 (b). As for the LEDs, near infra-red $850 \mathrm{~nm}$ LEDs were chosen for their deep tissue penetration characteristics and the availability of high-powered products. The LED chips were mounted in an array. Each LED array had a $50 \mathrm{~mm}$ x $7 \mathrm{~mm}$ aperture, pulse width $70 \mathrm{~ns}$, and pulse energy $200 \mu \mathrm{J} /$ pulse per array totaling $400 \mu \mathrm{J} /$ pulse for 2 arrays.
The positioning of the ultrasound probe and LED arrays were such that the LED arrays were placed on both sides of the probe closer to the sample than the transducer at the tip of the ultrasound probe. The distance between the LED arrays was as narrow as possible to allow the light to be emitted to the area directly beneath the probe.

The LED arrays were synchronized by the timing signal of a controller $\mathrm{PC}$ at a rate of $4 \mathrm{kHz}$ and as shown in the drive circuit of the LEDs (Figure Figure 2 (c)), the low on-resistance MOSFET (Metal-Oxide-Semiconductor Field-Effect Transistor) allows $r$ the LED to be switched on and off at an extremely high rate according to the pulse signal input. At each on-off signal a $400 \mathrm{~V}$ pulse was applied, creating a pulse current to the LED array, and as a result pulsed light was emitted. Under these conditions the halfwidth resolution of a human hair was $268 \mu \mathrm{m}$ deep and a line/space of $0.59 \mathrm{~mm}$ was able to be distinguished laterally ${ }^{12)}$

As for the phantom sample shown in Figure 1, bovine blood in a micro-test tube as shown in Figure Figure 2(d) was used. The tube filled with blood was placed $21 \mathrm{~mm}$ away from the transducer of the ultrasound probe all of which is submerged beneath the surface of the intralipid. Since the focal distance from the rubber lens of the ultrasound probe was $15 \mathrm{~mm}$ and the sensitivity was maximum at this distance, the sample was placed to be as near as possible to this distance while still being near the center of the image. Scanning of the sample was performed on the cross section of the micro-test tube containing the blood as shown by the red line in Figure $\mathbf{2}(\mathbf{d})$, and photoacoustic signals of this cross section were attained.

As shown in Figure 1, photoacoustic waves emitted from the bovine blood were detected by the ultrasound probe with a128 ch transducer and were converted to electric signals which were then amplified for a gain of $40-100 \mathrm{~dB}$. Parallel and simultaneous amplification of signals from $128 \mathrm{ch}$ requires a multi-channel amplifier IC

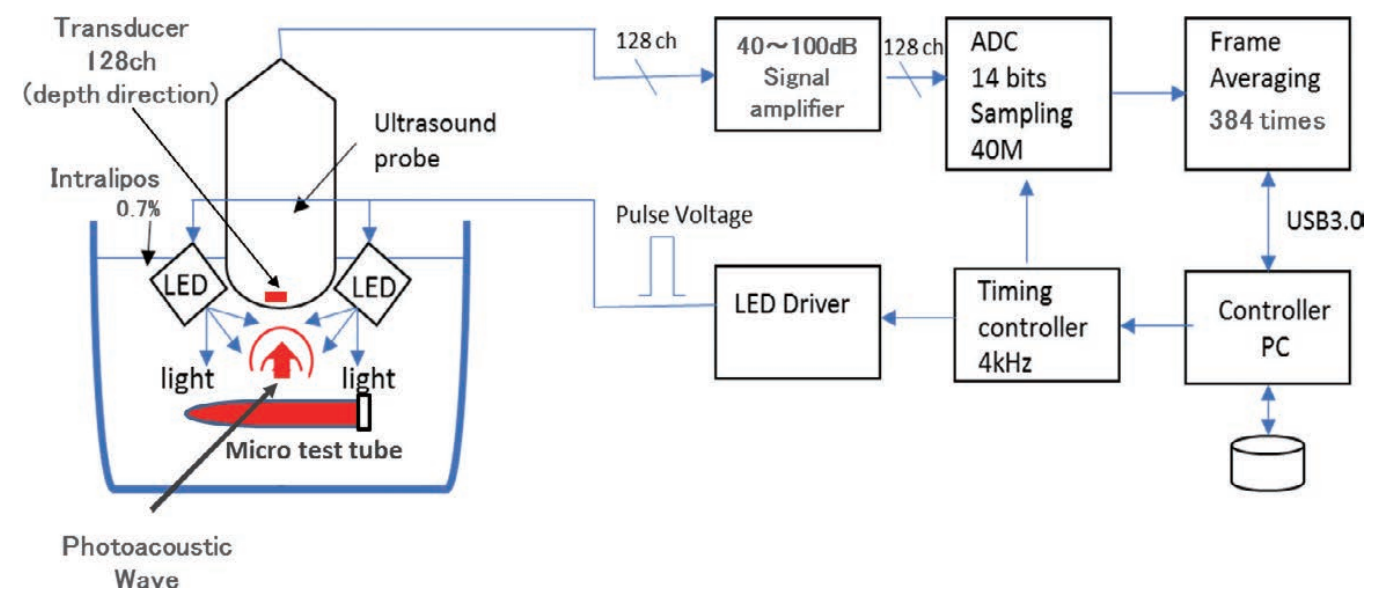

Fig. 1: Experimental setup. 
(in this case $8 \mathrm{ch}$ ). However, presently available multi-channeled amplifiers can only achieve a maximum gain of $50 \mathrm{~dB}$, and therefore in order to achieve a total gain of $100 \mathrm{~dB}$, 2-step amplification with 2 units was required. The set-up of the circuit model is shown in Figure 3. Each signal amplifier achieved a gain of A and a total gain AxA was reached with the 2 signal amplifiers. As an example, if a total gain of $80 \mathrm{~dB}$ for the whole system was required, both the front and rear units were set the same at $40 \mathrm{~dB}$ per unit. The signals were then converted by a 14 bit $40 \mathrm{MHz}$ sample analog-digital converter (ADC) to 1024 digital signals in the vertical (depth) direction (in water this amounts to approximately $38.4 \mathrm{~mm}$ ).
The created image was a pre-image reconstruction with a matrix size $128 \times 1024$ (Rf-raw data image) as shown inFigure Figure 2(f). This image was sent to an exclusive PC through a USB 3.0 interface where averaging took place 384 times and by comparison to the signal, random noise was relatively reduced ${ }^{13}$. Following this process, image reconstruction operation and image output processing took place and the final image wass visualized on the PC monitor.

Images of bovine blood were taken with an amplifier gain of $40 \mathrm{~dB}$ to $100 \mathrm{~dB}$, in $10 \mathrm{~dB}$ increments. The reconstructed image is shown in Figure 4. The size of the image was $38.4 \mathrm{~mm}$ wide (128 ch direction) and $38.4 \mathrm{~mm}$
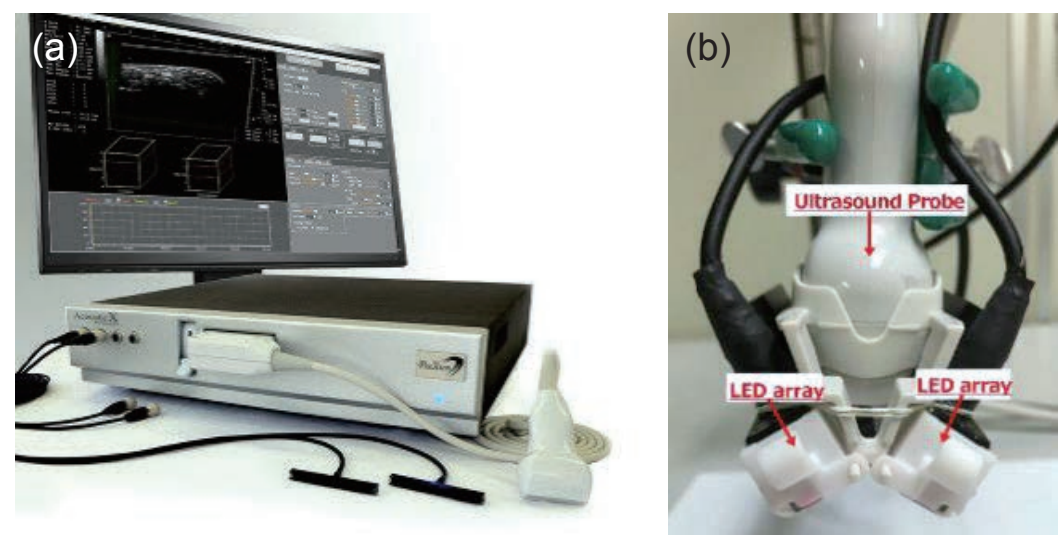

(c)

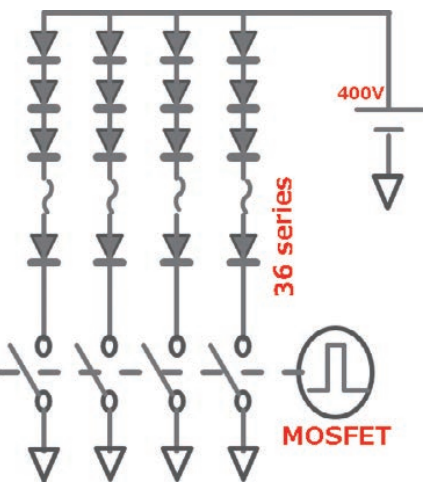

(d)

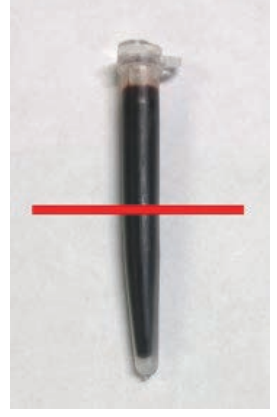

(e)

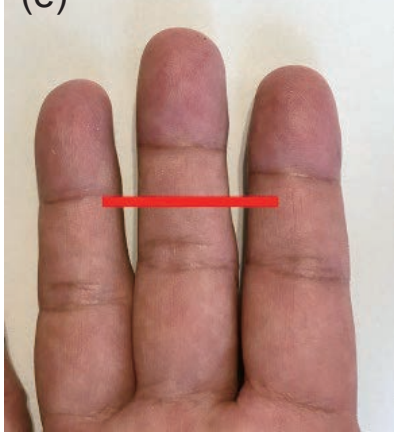

(f)

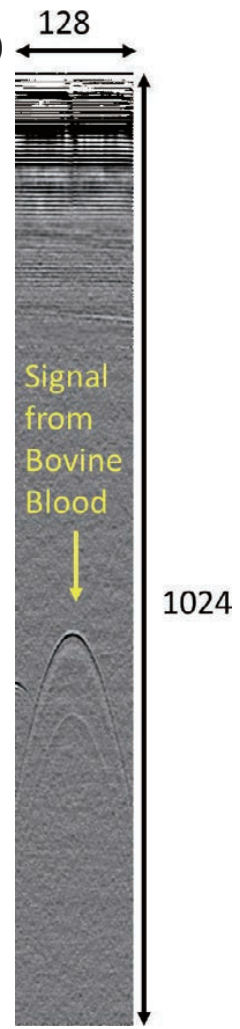

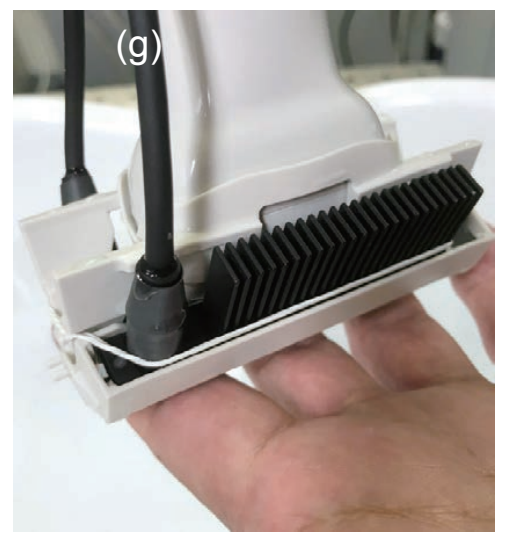

Fig. 2: (a) Photoacoustic Imaging System AcousticX (CYBERDYNE;Tokyo,Japan). (b) LED arrays and Ultrasound probe. (c) Drive circuit of LEDs. (d) Bovine Blood in Micro Test Tube and Scan Direction (solid line). (e) Human Fingers and Scan Direction (solid line). (f) Example of Rf- raw data image. (g) Arrangement of Scanned Human Fingers and Ultrasound probe with LED arrays. 


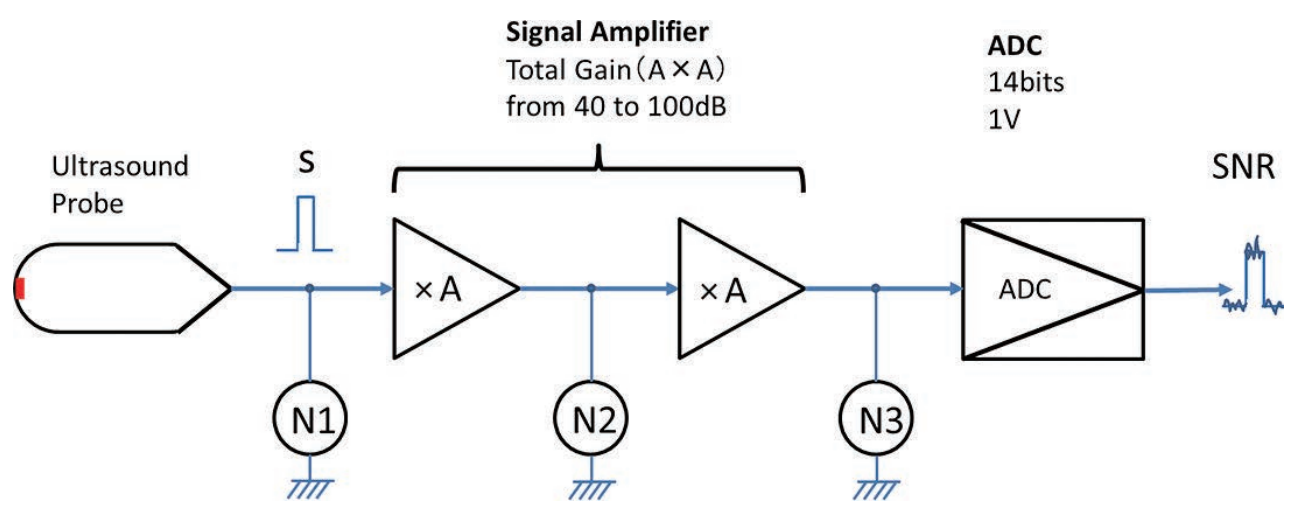

Fig. 3: Circuit model for SNR simulation.

deep. In this image, the photoacoustic signal from the bovine blood is depicted as a point. In order to calculate the SNR, the following method was used to attain noise (N) and signal (S). Firstly, an area of 50 x 50 pixels (actual size $4.5 \mathrm{~mm} \times 4.5 \mathrm{~mm}$ ) to the right of the photoacoustic signal was chosen for evaluation. The standard deviation $(\sigma)$ of the data within this area was calculated and the peak value $\pm 3 \sigma$ containing $99.7 \%$ of the data was defined to be noise (N). As for the photoacoustic signal (S), the maximum value was used, but since it can be assumed that the true value of $\mathrm{S}$ would include half of $\mathrm{N}$ $(3 \sigma)$, therefore $3 \sigma$ was subtracted from the maximum value and was defined as $S$.

\subsection{Measurements of photoacoustic signals, noise and the evaluation of the image for the photoacoustic imaging of blood vessels of a human finger}

In compliance to the Helsinki Declaration, written informed consent was attained from the human volunteer subject for this experiment. The same system was used to detect photoacoustic signals adjusting the amplifier gain from $40 \mathrm{~dB}$ to $100 \mathrm{~dB}$, in $10 \mathrm{~dB}$ increments. The set-up was the same as in Figure 1 where the bovine blood micro-test tube was replaced with human fingers and the photoacoustic imaging was performed (Figure 2(e)). The skin surface of the finger was submerged in the intralipid and was placed $10 \mathrm{~mm}$ deep from the transducer of the ultrasound probe. This distance was set to be as near as possible to the distance of maximum sensitivity of the ultrasound probe, which is $15 \mathrm{~mm}$, and close enough to the LED arrays so that light attenuation would not become an issue. The positioning of the ultrasound probe and fingers submerged in intralipid is shown in Figure 2(g). The solid red line in Figure 2(e) shows the area of the ultrasound scan, from the index finger, middle finger and ring finger. The main area, roughly halfway between the distal and proximal interphalangeal joints of the middle finger, was scanned and photoacoustic signals were obtained. In this photoacoustic imaging, the signals were released mainly from the hemoglobin inside the blood vessels, and the image acquired shows cross sections of subcutaneous veins and capillaries (Figure 5(a)).

The ultrasound image obtained simultaneously (Figure 5(b)) shows the skin and subcutaneous tissue, however bone existed 2-3 $\mathrm{mm}$ deep and since the acoustic impedance of bones differs greatly than that of soft-tissue and blood vessels, the image deeper than this contained many artifacts. The photoacoustic imaging data of Figure 5(a) was converted from white to red and superimposed on the ultrasound image (Figure 5(b)) to create Figure 5(c) where the relative positional relationship could be discerned.

For the calculation of the SNR, the maximum signal of the yellow line in Figure 5(d) was deemed $S$ while the standard deviations ( $\sigma$ ) for a 50 x 50 pixel area in the intralipid just above the area of the signal was calculated with the same method as in the bovine blood for the determination of $\mathrm{N}$.

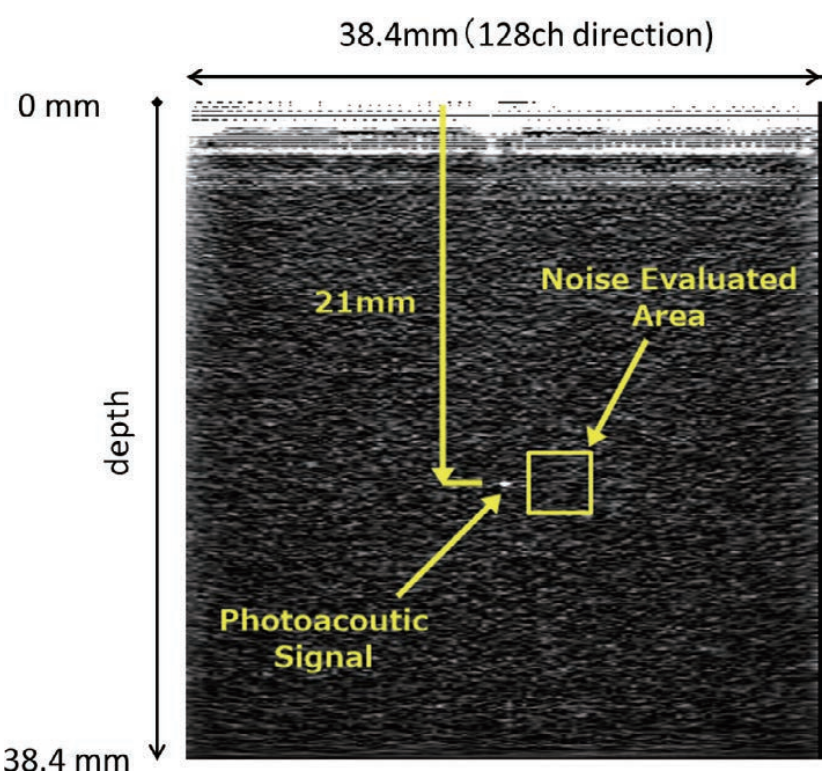

Fig. 4: Photoacoustic signal and noise evaluated area. 


\section{Results}

Table 1 shows the values of $S, N$ and the SNR from the photoacoustic imaging of bovine blood with an amplifier gain of 40 to $100 \mathrm{~dB}$ in $10 \mathrm{~dB}$ increments. A scatter dia- gram manifesting the amplification dependency of the SNR is shown in Figure 6. The actual photoacoustic images of bovine blood at the respective amplifier gains are shown in Figure $\mathbf{7 ( a ) - ( g ) . ~ T h e ~ c e n t e r ~ o f ~ t h e ~ i m a g e s ~ i s ~}$ the photoacoustic signals and the size of the images is 50 (a)

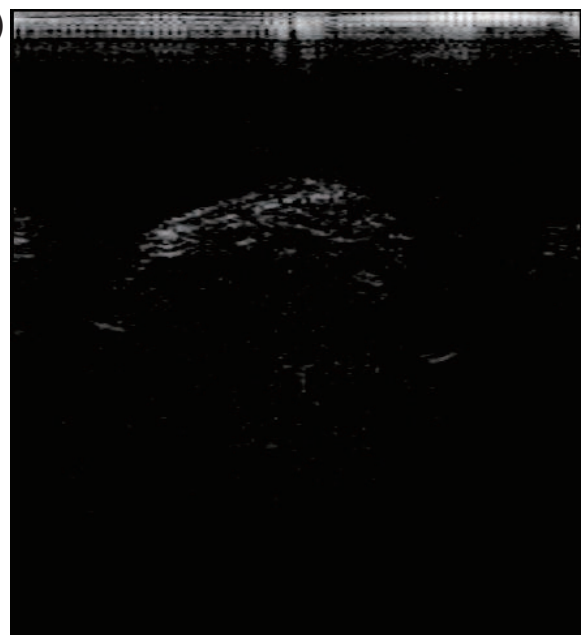

(c)

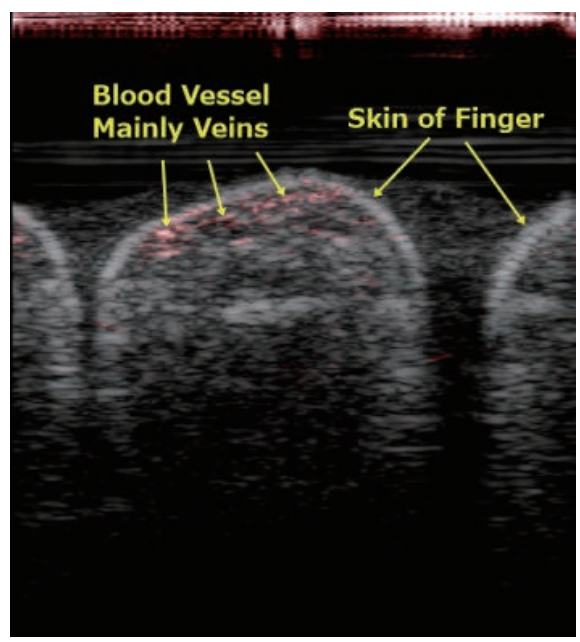

(b)

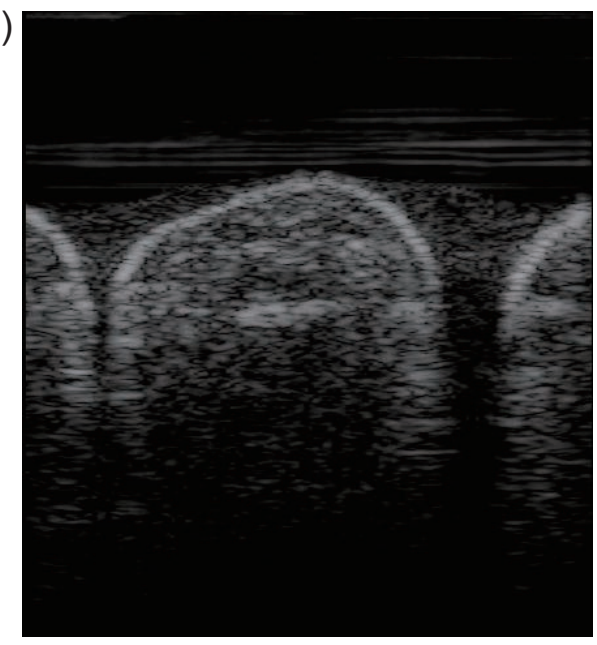

(d)

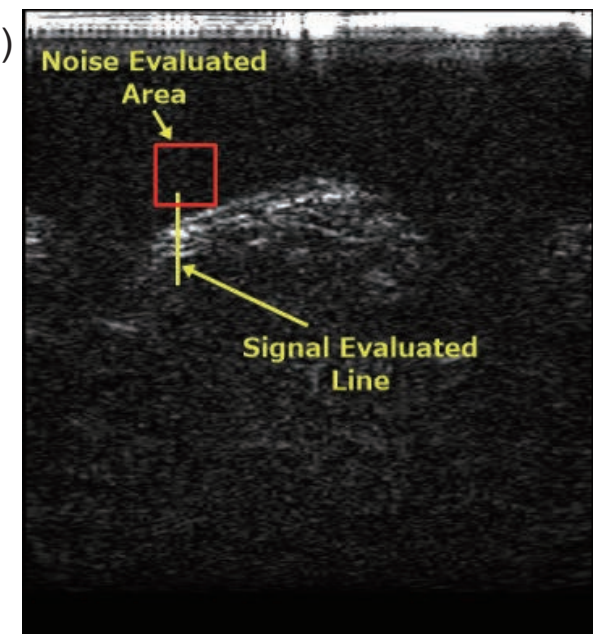

Fig. 5: (a) Photoacoustic image data of human finger (grayscale). (b) Ultrasound image data of human finger (grayscale). (c) Photoacoustic (red) and Ultrasound (grayscale) image data of human finger. (d) Photoacoustic signal evaluated line (yellow) and noise evaluated area (red).

Table 1: Signal, Noise and calculated SNR of bovine blood at a total gain of from $40 \mathrm{~dB}$ to $100 \mathrm{~dB}$.

\begin{tabular}{cccc}
\hline Total Gain (dB) & Signal (digit) & Noise (digit) & SNR \\
40 & 1.06 & 3.00 & 0.35 \\
50 & 3.54 & 4.14 & 0.85 \\
60 & 15.30 & 7.04 & 2.17 \\
70 & 54.50 & 13.60 & 4.01 \\
80 & 189.16 & 29.10 & 6.50 \\
90 & 631.19 & 67.64 & 9.33 \\
100 & 1622.70 & 165.91 & 9.78 \\
\hline
\end{tabular}

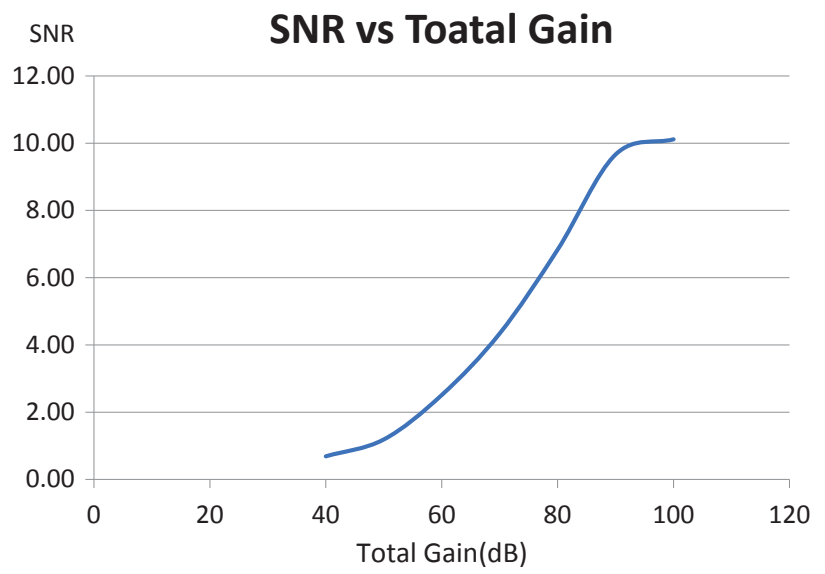

Fig. 6: SNR vs Total Gain when using human blood. 
(a)

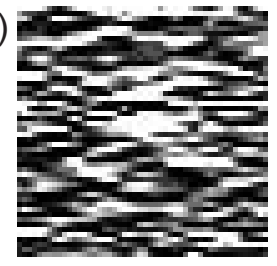

(e)

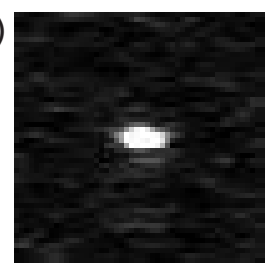

(b)

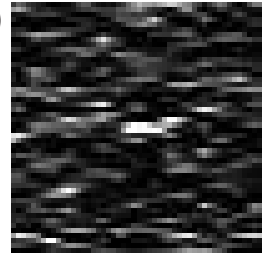

(f)

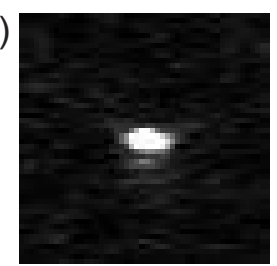

(c)

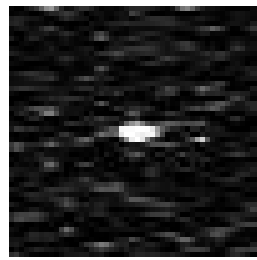

(g)

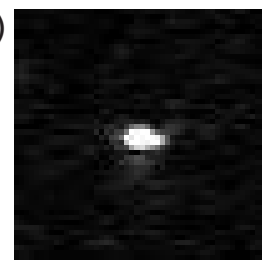

(d)

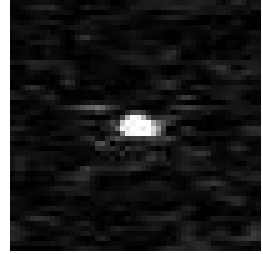

Fig 7: Photoacoustic signal image of bovine blood in amplifier gain of (a) at $40 \mathrm{~dB}$, (b) at $50 \mathrm{~dB},(\mathrm{c})$ at $60 \mathrm{~dB},(\mathrm{~d})$ at $70 \mathrm{~dB},(\mathrm{e})$ at $80 \mathrm{~dB},(\mathrm{f})$ at $90 \mathrm{~dB},(\mathrm{~g})$ at $100 \mathrm{~dB}$.

Table 2: Signal, Noise and calculated SNR of human finger blood at a total gain of from $40 \mathrm{~dB}$ to $100 \mathrm{~dB}$.

\begin{tabular}{cccc}
\hline Total Gain $(\mathrm{dB})$ & Signal (digit) & Noise (digit) & SNR \\
\hline 40 & 1.24 & 2.89 & 0.43 \\
50 & 3.02 & 4.96 & 0.61 \\
60 & 14.84 & 10.67 & 1.39 \\
70 & 55.18 & 19.13 & 2.88 \\
80 & 172.28 & 38.92 & 4.43 \\
90 & 672.90 & 102.00 & 6.60 \\
100 & 1817.40 & 238.90 & 7.61 \\
\hline
\end{tabular}

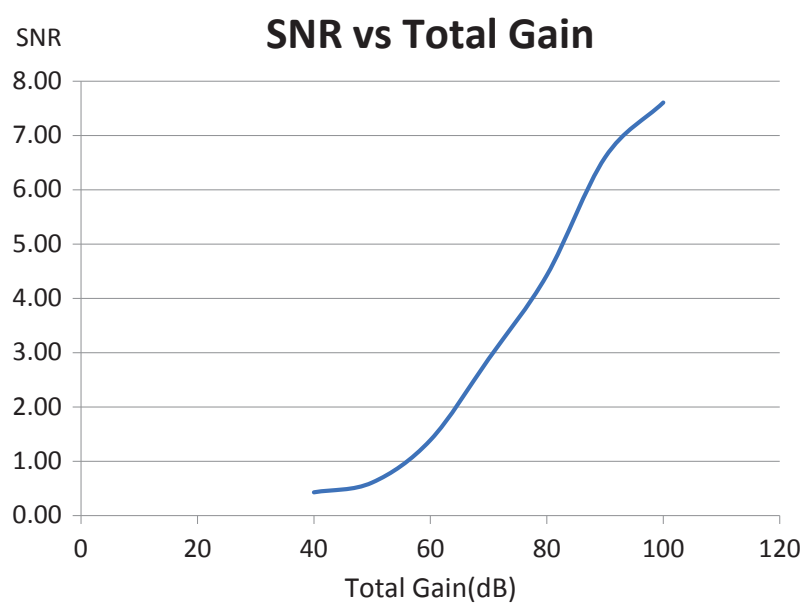

Fig. 8: SNR vs Total Gain when using human blood.

x 50 pixels (actual size $4.5 \mathrm{~mm}$ x $4.5 \mathrm{~mm}$ ). From Table 1, the SNR was 0.35 at an amplifier gain of $40 \mathrm{~dB}$. As the amplifier gain was increased by $10 \mathrm{~dB}$, the SNR also increased to where the SNR at an amplifier gain of $90 \mathrm{~dB}$ was 9.33 and at an amplifier gain of $100 \mathrm{~dB}$ was 9.78, showing a tendency towards saturation. As seen in Figure 7(a)-(g), visual recognition of the photoacoustic signal was barely discernible at the amplifier gain of 40 $\mathrm{dB}$, whereas at $50 \mathrm{~dB}$ the signal was readily recognizable.

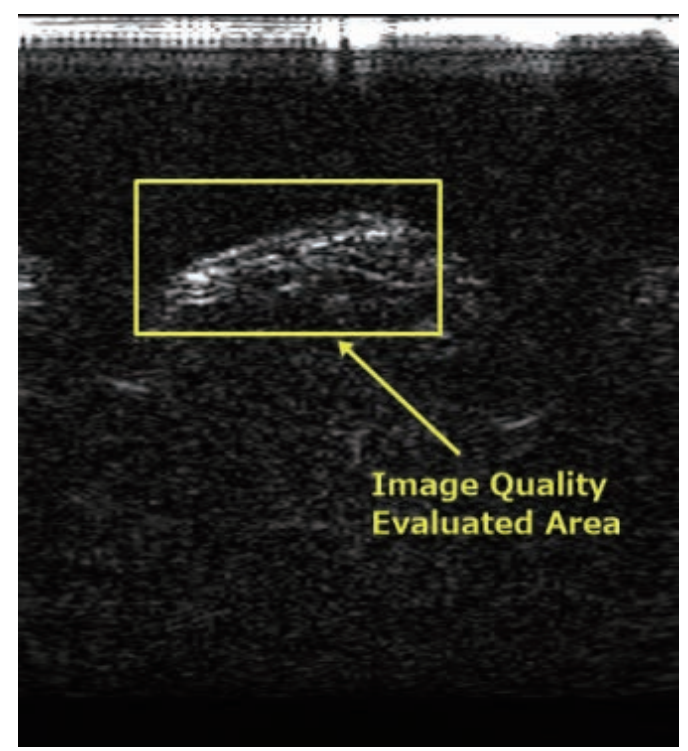

Fig 9: Photoacoustic image data area (yellow) for image quality evaluation.

As the amplifier gain increased the signal intensity increased while the surrounding noise decreased inversely. The size of the signal increased in proportion to the increase of the amplifier gain, but after the amplifier gain of $70 \mathrm{~dB}$, the change in size became minute.

Table 2 shows the values of S, N and the SNR for the photoacoustic imaging of the human fingers with an amplifier gain of 40 to $100 \mathrm{~dB}$ at $10 \mathrm{~dB}$ increments, while Figure 8 shows the dependence of SNR on the amplifier gain. The image evaluation area is shown as a rectangular box in Figure 9. The area is 200 pixels x 100 pixels (actual size, $18 \mathrm{~mm}$ x $9 \mathrm{~mm}$ ). Actual photoacoustic images are shown in Figure 10 (a)-(g). From Table 2, the SNR was 0.43 at amplifier gain $40 \mathrm{~dB}$, and as the amplifier gain increased SNR also increased reaching 7.61 at amplifier gain $100 \mathrm{~dB}$. Visual recognition of the signal, was undiscernible at amplifier gain $40 \mathrm{db}$ where the signal was completely buried in the surrounding noise, as seen in 
(a)

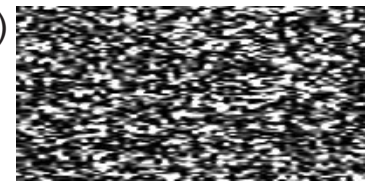

(d)

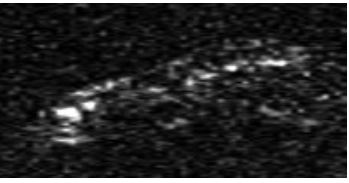

(g)

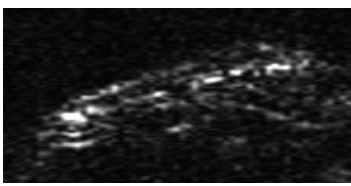

(b)

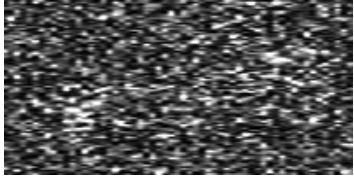

(e)

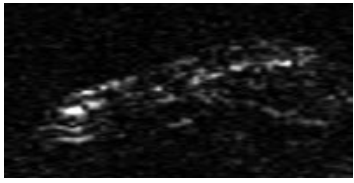

(c)

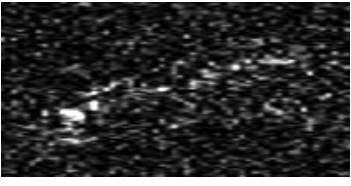

(f)

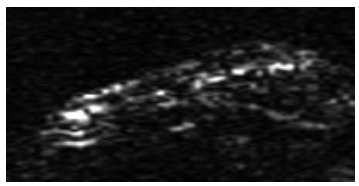

Fig 10: Finger blood image in amplifier gain of (a) at $40 \mathrm{~dB}$, (b) at $50 \mathrm{~dB}$, (c) at $60 \mathrm{~dB}$, (d) at $70 \mathrm{~dB},(e)$ at $80 \mathrm{~dB},(\mathrm{f})$ at $90 \mathrm{~dB},(\mathrm{~g})$ at $100 \mathrm{~dB}$.
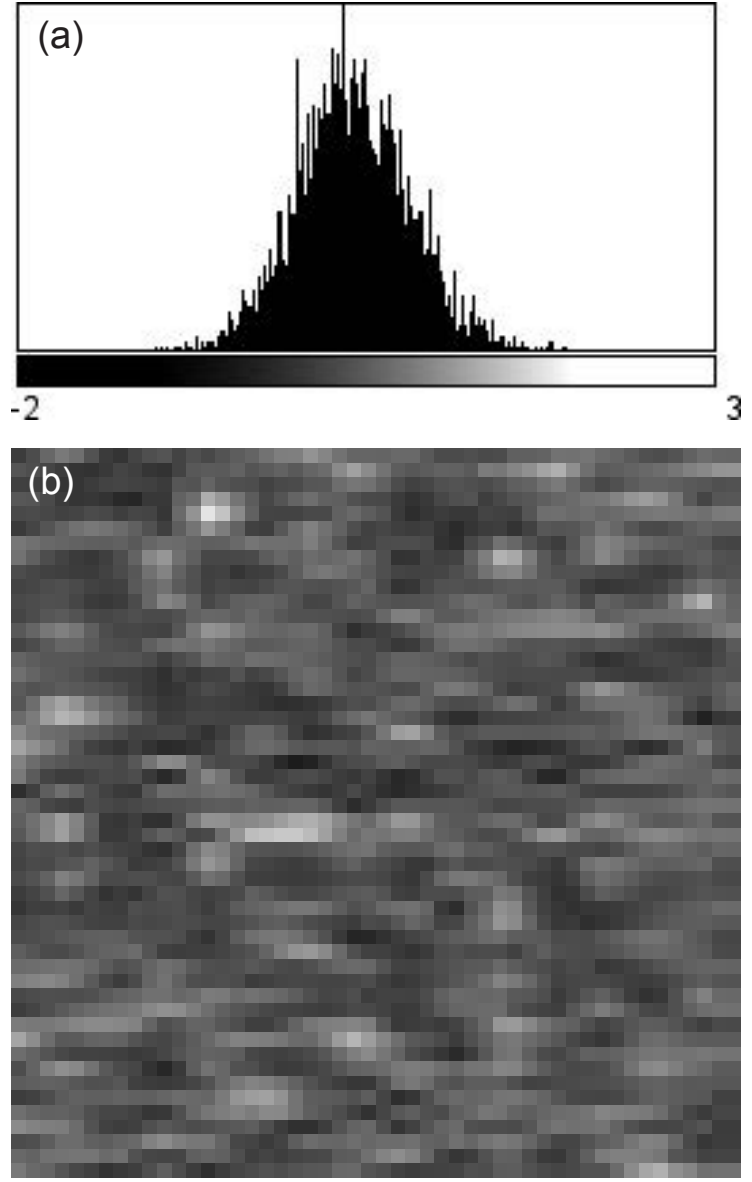

Fig 11: FHistogram (a) and image (b) of noise evaluated area at $40 \mathrm{~dB}$ gain.

Figure 10(a). The signal is barely noticeable at $50 \mathrm{~dB}$ but as the amplifier gain increased the noise decreased, and distribution of the blood vessels become more and more clear. After the amplifier gain of $80 \mathrm{~dB}$, improvements in the image clarity decreased. Although there was a tendency that more subtle structures could be visualized at higher amplifier gains, the differences was not statistically significant.

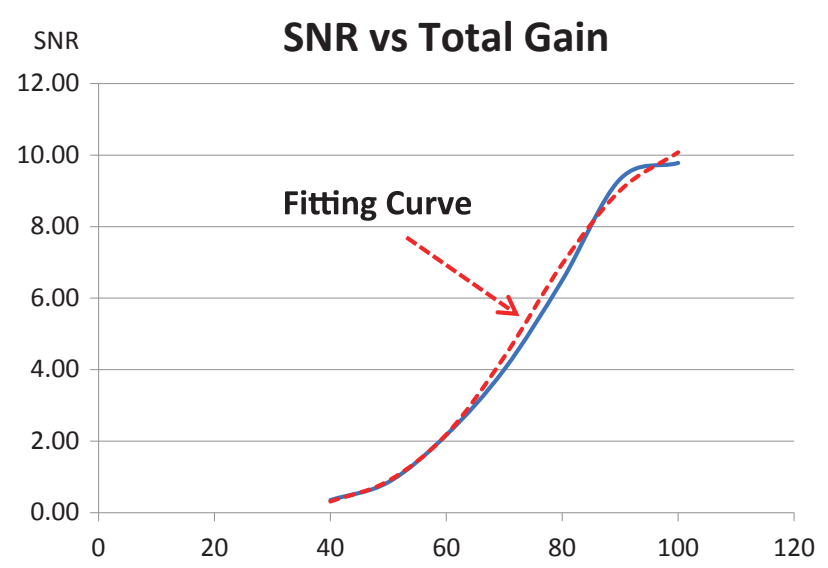

Fig. 12: Curve fitting for SNR of bovine blood experiment.

\section{Discussion}

The relationship between signal and noise in the signal processing circuit of a photoacoustic imaging system, where the detected signals from the transducer of the ultrasound probe are amplified in a two-step fashion (front and rear) and the amplified signal is converted from analog to digital, can be explained by a circuit model as shown in Figure 3. The signal (s), detected by the transducer of the ultrasound probe is amplified A times in the front amplifier and then is amplified again at the rear amplifier for further A times for a total amplification of A $\mathrm{x}$ A. Noise, on the other hand, can be categorized into three types. First is noise amplified A x A by both front and rear amplifiers, the same as (s), (N1). Second is noise amplified just once at the rear amplifier for an amplification of A times (N2) and the third is noise fixed and unaffected by the amplifiers (N3). Such noise can be considered random as shown in the histogram (Figure 11 (a)) and image (Figure 11(b)) of the noise evaluation at 40 $\mathrm{dB}$ gain. In order to evaluate the size of the noise in relation to the signal, the energy of the noise (amounting to 
the squared value of the noise) can be summed and used. Therefore, in this circuit model the SNR can be expressed as the following equation.

$$
\mathrm{SNR}=\frac{\mathrm{s} \times \mathrm{A} \times \mathrm{A}}{\sqrt{(\mathrm{N} 1 \times \mathrm{A} \times \mathrm{A})^{2}+(\mathrm{N} 2 \times \mathrm{A})^{2}+\mathrm{N}^{2}}}
$$

From the curve of Figure $\mathbf{6}$ and curve fitting using the above equation, the values of N1-N3 as multiples of (s) were, N1 $=0.935 \times$ s, N2 $=10.5 \times$ s, N3 $=300 \times$ s. The curve fitted SNR of Figure $\mathbf{6}$ superimposed on the actual measured values of Figure $\mathbf{6}$ is shown in Figure 12. When the values of N1-N3 attained from the fitted curve were assigned to the equation, the $\operatorname{SNR}(\mathrm{A} \rightarrow \infty)=$ $1 / 0.0935=10.70$. This means that no matter how much the amplifier gain is increased, the SNR will be a ratio of the output signal (s) from the transducer of the ultrasound probe and noise (N1) and will reach saturation at $\mathrm{SNR}=10.70$.

When a photoacoustic imaging system using solid-state lasers as the light source is used, the photoacoustic signal is large due to the extremely high peak power from a laser, compared to the photoacoustic signal of an LED-based system. Therefore, only a single step, single amplifier with a gain of $40 \mathrm{~dB}$, as seen in generally marketed ultrasound diagnostic devices, is required. The circuit model in such systems would be such as the model of Figure 3 without the rear amplifier. In this case the SNR can be expressed as the equation shown below.

$$
\mathrm{SNR}=\frac{\mathrm{s} \times \mathrm{A}}{\sqrt{(\mathrm{N} 1 \times \mathrm{A})^{2}+\mathrm{N}^{2}}}
$$

When the amplifier gain is $40 \mathrm{~dB}$, meaning 100 times, this number is assigned as A, while N1 and N3 attained from curve fitting is substituted in the equation, the value of the SNR can be predicted. The result would be $\mathrm{SNR}=0.33$, and matching images such as those in Figure 7(a) and Figure 10(a) would be useless.

\section{References}

1: Kim C, Song KH, Gao F, et al. (2010): Sentinel lymph nodes and lymphatic vessels: Noninvasive dual-modality in vivo mapping by using indocyanine green in rats-Volumetric spectroscopic photoacoustic imaging and planar fluorescence imaging, Radiology,255: 442-450.

2: Garcia-Uribe A, Erpelding TN, Krumholz A, et al. (2015): Dual-modality photoacoustic and ultrasound imaging system for noninvasive sentinel lymph node detection in patients with breast cancer, Sci Rep, 5:15748.

3: Wang X, Xie X, Ku G, et al. (2006): Noninvasive imaging of hemoglobin concentration and oxygenation in the rat brain using high-resolution photoacoustic tomography, J Biomed Opt, 11: 024015.

4: Piras D, Grijsen C, Schutte P, et al. (2013): Photoacoustic needle: minimally invasive guidance to biopsy, J Biomed Opt, 18: 070502

5: Yao Q, Ding Y, Liu G, et al. (2017): Low-cost photoacoustic
The SNR from the venous blood of a human finger is shown in Table 2 and Figure 8 . The SNR tended to increase as the gain was increased. The SNR of 7.94 at gain of $100 \mathrm{~dB}$ was somewhat smaller than that of bovine blood (SNR $=9.78)$ but the difference was small and discussion comparing the two is possible. In Figure 10(a)-(g), the image at a gain of $70 \mathrm{~dB}$ still leaves a noisy impression. However, at gains over $80 \mathrm{~dB}$ the effect of noise decreased and even though clarity of the images somewhat improved reaching the gain of 100 $\mathrm{dB}$, the image at gain of $80 \mathrm{~dB}$ was thought to suffice for practical purposes as a photoacoustic image. The SNR at this level was 4.43 .

In Figure 7(a)-(g), the image of bovine blood became stable at a gain of $70 \mathrm{~dB}$. The SNR at that point was 4.01 from Table 1. Stable images of both bovine blood and human blood vessels were attained at coinciding SNRs of over 4. This means that for practical purposes, photoacoustic images with an SNR greater than 4 are required.

\section{Conclusion}

In this study, the authors demonstrated that in order to create real time photoacoustic images of human blood using an LED-based photoacoustic image system, an amplifier with a gain of over $80 \mathrm{~dB}$ is required and SNR $>4$ must be achieved. If a more detailed, clearer image is required increasing the gain to $100 \mathrm{~dB}$ would be better. The Accoustic X system which incorporates an amplifier with a gain of $97 \mathrm{~dB}$ is therefore sufficient for real time photoacoustic imaging.

This study also discussed the difficulty of real time photoacoustic imaging using LED arrays as the light source and detection by solid-state laser based photoacoustic imaging systems for the low amplification of a mere $40 \mathrm{~dB}$. imaging systems based on laser diode and light-emitting diode excitation, J Innov Opt Health Sci,10: 1730003.

6: Allen TJ, Beard C (2016): High power visible light emitting diodes as puled excitation sources for biomedical photoacoustics, Biomed Opt Express, 7: 1260-1270.

7: Agano T, Sato N (2016): Photoacoustic imaging system using LED light source, Conf Lasers Electro Optics: ATh 3 N.5.

8: Agano T, Sato N, Nakatsuka H, et al. (2015): Comparative experiments of photoacoustic system using laser light source and LED array light source, Proc SPIE, 9323: 93233X.

9: Agano T, Sato N, Nakatsuka H, et al. (2015): Attempts to increase penetration of photoacoustic system using LED array light source, Proc SPIE, 9323: 93233Z.

10: Agano T, Sato N, Nakatsuka H, et al. (2016): High frame rate photoacoustic imaging using multiple wave-length LED array light sources, Proc SPIE,9708: 97084E.

11: Zhu Y, Xu G, Yuan J, et al. (2018): Light emitting diode 
ORIGINAL ARTICLES

based photoacoustic imaging and potential clinical applications, Sci Rep,8: 9885.

12: Hariri A, Lemaster J, Wang J, et al. (2018): The characterization of an economic and portable LED-based photoacoustic imaging system to facilitate molecular imaging, Photoacous- tics, 9: 10-20.

13:Agano T, Sato N (2017): Photoacoustic signal detection using LED light source, RIEC International Workshop on Biomedical Optics 2017: OQD-17-017.

\section{Acknowledgement}

The authors thank Mr. Naoto Satoh from CYBERDYNE Inc. for his apt advices in conducting this investigation.

Disclosure of conflicts of interests

No conflict of interest exists. 